\title{
Usefulness of the Doppler Mean Gradient in Evaluation of Children with Aortic Valve Stenosis and Comparison to Gradient at Catheterization
}

\author{
A. Resai Bengur, MD, A. Rebecca Snider, MD, Gerald A. Serwer, MD, Jane Peters, \\ and Amnon Rosenthal, MD
}

To assess the usefulness of the Doppler mean gradient as a noninvasive indicator of the need for intervention, 33 children (ages 3 months to 20 years) with valvular aortic stenosis (AS) underwent a 2-dimensional and Doppler echocardiographic examination a median of $\mathbf{1}$ day before cardiac catheterization. The clinical decision for intervention was based on finding a catheterization peak-to-peak pressure gradient of $>75 \mathrm{~mm} \mathrm{Hg}$ or from 50 to 75 $\mathrm{mm} \mathrm{Hg}$ in the presence of symptoms or an abnormal exercise treadmill test result. Of the 33 patients, 23 required intervention. The decision for intervention was compared to the Doppler mean gradient, and the Doppler peak and mean gradients were compared to the catheterization peak-to-peak gradient. All 12 patients with a Doppler mean gradient $>27 \mathrm{~mm} \mathrm{Hg}$ had intervention and had a catheterization peak-to-peak gradient of $\geq 75 \mathrm{~mm} \mathrm{Hg}$. All 3 patients with a Doppler mean gradient $<17$ $\mathrm{mm} \mathrm{Hg}$ had no intervention and had a peak-to-peak gradient <50 mm Hg. The remaining 18 patients with Doppler mean gradlents between 17 and 27 mm Hg comprised an intermediate group in whom the Doppler mean gradient alone did not predict the need for intervention.

From a chi-square table, a Doppler mean gradient $>27 \mathrm{~mm} \mathrm{Hg}$ predicted the need for intervention with $100 \%$ specificity (no false positives) and $52 \%$ sensitivity (11 false negatives). If a Doppler mean gradient $>\mathbf{2 4} \mathrm{mm} \mathrm{Hg}$ was used to predict intervention, the sensitivity increased to $91 \%$ (2 false negatives) but specificity decreased to $70 \%$ ( 3 false positives). To improve the ability to predict the need for intervention in patients with a Doppler mean gradient between 17 and $27 \mathrm{~mm} \mathrm{Hg}$, the presence of symptoms or an abnormal exercise treadmill test result was combined with the Doppler mean gradient as criteria for intervention. When the criteria for intervention were a Doppler mean gradient $>27 \mathrm{~mm} \mathrm{Hg}$ or a Doppler mean gradient from 17 to $27 \mathrm{~mm} \mathrm{Hg}$ in the presence of symptoms or an abnormal exercise test, sensitivity was $96 \%$ (1 false negative) and specificity was $80 \%$ (2 false positives). Catheterization peak-to-peak gradients correlated well with Doppler mean and peak gradients ( $r=0.74$ and 0.73 , respectively).
Thus, the Doppler mean gradient is a useful indicator of the need for intervention in children with AS. A Doppler mean gradient $>27 \mathrm{~mm} \mathrm{Hg}$ indicates the need for intervention with $\mathbf{1 0 0} \%$ specificity while a Doppler mean gradient $<17 \mathrm{~mm}$ Hg predicts mild AS. For patients with Doppler mean gradient between 17 and $27 \mathrm{~mm} \mathrm{Hg}$, additional noninvadive data are necessary to determine the need for intervention.

(Am J Cardiol 1989;64:756-761)

I n children with valvular aortic stenosis (AS), the peak-to-peak pressure gradient measured at cardiac catheterization is often used to determine the severity of AS and the need for intervention. Currently, the most widely used noninvasive technique for estimating the severity of AS is measurement of the peak instantaneous pressure gradient with Doppler echocardiography. ${ }^{1-4}$ The Doppler-derived peak instantaneous pressure gradient does not always correlate well with the catheterization-measured peak-to-peak pressure gradient. ${ }^{4-6}$ Therefore, additional noninvasive estimates of the severity of AS would be helpful in making patient management decisions. The Doppler mean gradient is another noninvasive measurement that has proven useful in the evaluation of adult patients with AS. . $^{5-12}$ In pediatric patients, little information is available correlating the Doppler mean gradient with the hemodynamic severity of the AS. This study assesses the usefulness of the Doppler mean gradient in predicting the need for intervention in children with AS.

\section{METHODS}

Patient population: All children with AS who underwent 2-dimensional and Doppler echocardiographic examination and cardiac catheterization at this institution between September 1984 and August 1988 were included in the study. The study population consisted of 33

From the Department of Pediatrics, C.S. Mott Children's Hospital, University of Michigan Medical Center, Ann Arbor, Michigan. Manuscript received February 6, 1989; revised manuscript received and accepted June 30, 1989.

Address for reprints: A. Rebecca Snider, MD, F1609, C.S. Mott Children's Hospital, University of Michigan, Ann Arbor, Michigan 48109-0204. 
patients whose ages ranged from 3 months to 20 years (10 \pm 6 years, mean \pm standard deviation) and whose weights ranged from 5 to $109 \mathrm{~kg}(41 \pm 29)$. Four infants $<3$ months old with critical AS were excluded from the study because critical AS in the neonatal period has different hemodynamic features and criteria for intervention. In addition, all children with subvalvular or supravalvular levels of left ventricular outflow obstruction were excluded.

The patients in the study group had several additional lesions. Aortic regurgitation was detected at cardiac catheterization in 17 patients. Regurgitation was graded at angiography as $1+$ in 12 patients, $2+$ in 4 and $3+$ in 1. Two children had previous repair of aortic coarctation with no residual descending aorta gradient at catheterization. Two patients had a small ventricular septal defect and 2 patients had mitral valve abnormalities including mitral valve prolapse in 1 and mild mitral stenosis in the other.

Echocardiographic examinations: In each patient, the 2-dimensional and Doppler echocardiographic examination was reviewed by 2 observers who had no knowledge of the results of catheterization or the patient's subsequent management. All echocardiographic examinations were performed from 1 to 90 days (median 1 day) before catheterization using either an Advanced Technology Laboratories Mark 600 or Ultramark 8 system or an Acuson 128 computed sonography system. Using high pulse repetition frequency or continuous wave Doppler techniques, the AS jet was recorded on videotape at 50 or $100 \mathrm{~mm} / \mathrm{s}$ sweep speed. The AS jet was recorded from apical, right parasternal and suprasternal transducer positions. The Doppler recording that provided the highest value for the peak velocity was used for subsequent measurements of the peak and mean gradients. The simplified Bernoulli equation (pressure gradient $=4 \times$ maximum velocity ${ }^{2}$ ) was used to calculate instantaneous pressure gradients. The peak instantaneous pressure gradient was defined as the largest of all the instantaneous gradients throughout systole and the mean gradient was calculated as the average of all the instantaneous gradients throughout systole. Using an off-line analysis system (Microsonics CAD 888), the gradients were calculated from a digital tracing of
FIGURE 1. Comparisons of Doppler mean (top) and peak (bottom) gradients with the catheterization peak-to-peak gradient in 33 children with aortic stenosis. The dotted ines represent the 5 and $\mathbf{9 5 \%}$ confidence intervals.

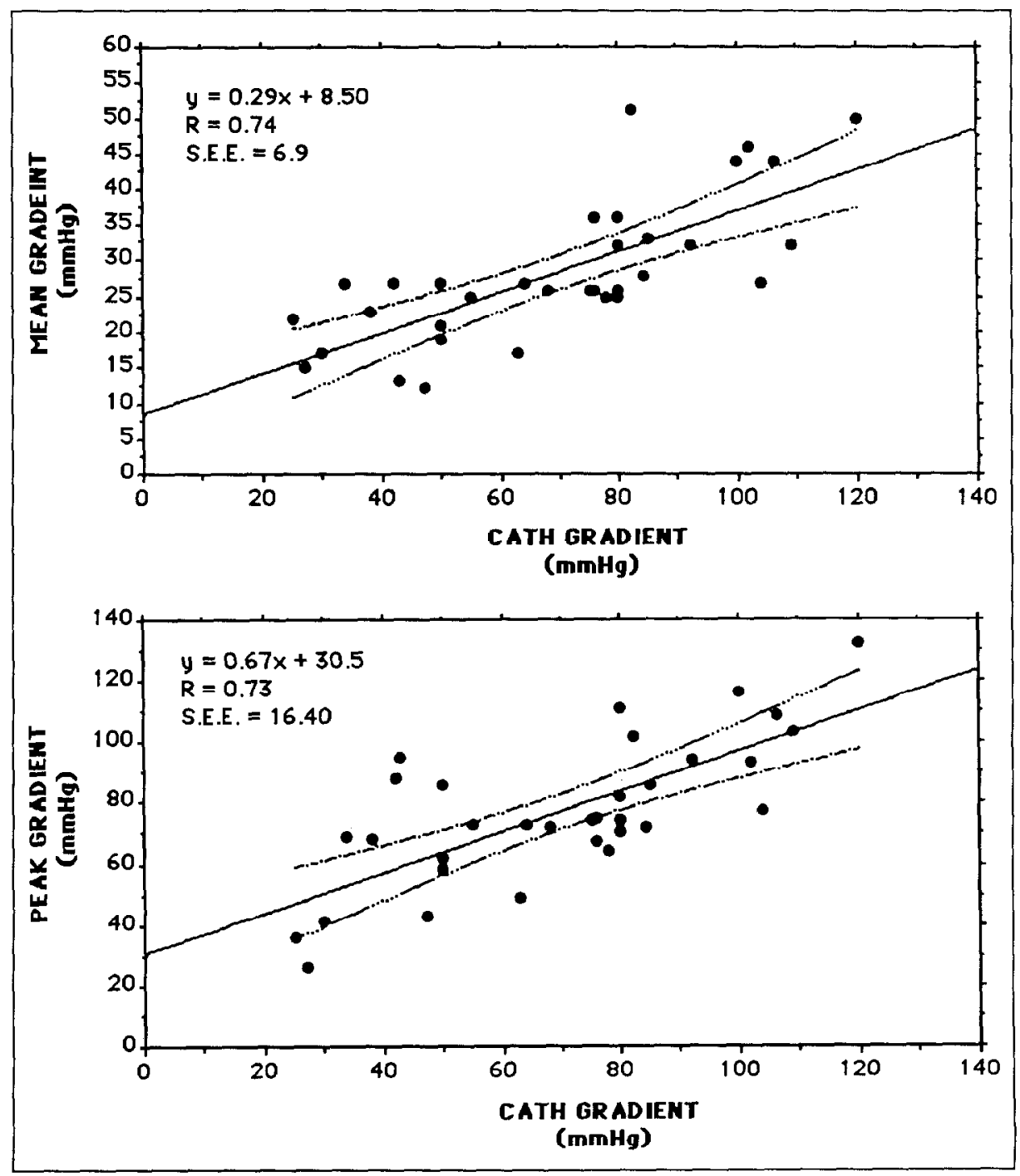


the outermost border of the Doppler spectral recording through systole. All data presented represent the average of 3 or more cardiac cycles.

Record review: The medical records of each patient were reviewed for the following information: (1) the medical history, with particular attention paid to the presence of symptoms including chest pain, syncope, dyspnea and easy fatigability; (2) results of exercise treadmill tests; and (3) results of cardiac catheterization, with peak-to-peak pressure gradient across the aortic valve, left ventricular end-diastolic pressure, thermodilution cardiac index and diagnosis of additional lesions obtained from the catheterization report.

Criteria for intervention: In our institution, the decision for intervention was based on finding a catheterization peak-to-peak gradient of $\geq 75 \mathrm{~mm} \mathrm{Hg}$ or from 50 to $75 \mathrm{~mm} \mathrm{Hg}$ in the presence of symptoms or an abnormal exercise treadmill test result. Using these criteria, 23 of the 33 patients required intervention. Twenty-two had balloon valvuloplasty and 1 had aortic valve replacement.

Statistical analysis: The sensitivity and specificity of different Doppler mean gradients for predicting the need for intervention were calculated from a chi-square table. Doppler mean and peak instantaneous pressure gradients were compared to catheterization-measured,

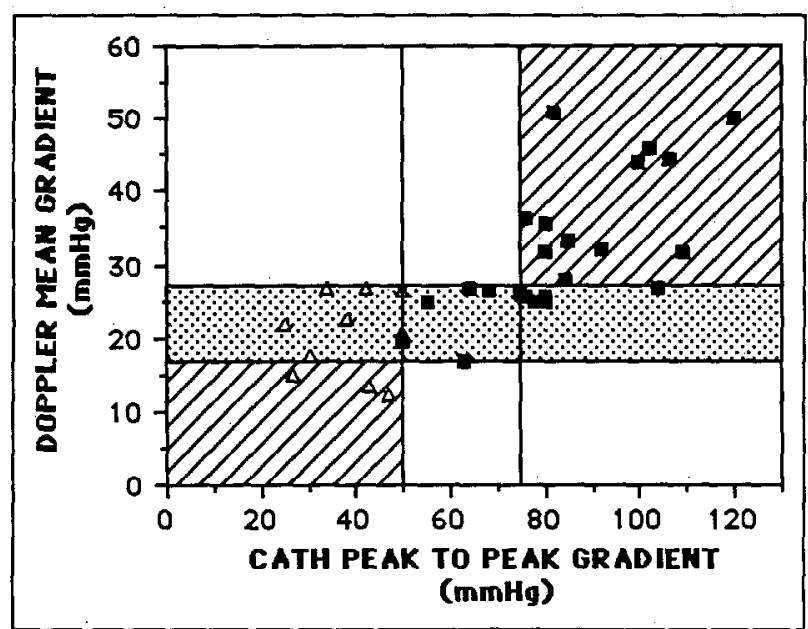

FICURE 2. Comparison of the Doppler mean gredient, the cathoterination pesk-to-peck gradient and the noed for intervention in 33 chidren with acric stenosis. Ch"litren who

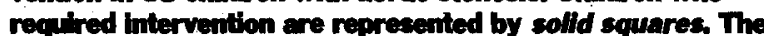
chilutron who dild not need intervention are depicted as open triangles The cross-hatched box on the upper right depicts the dita from 12 chiltren who recuired intervention and who hed a Doppler mean gradient $>\mathbf{2 7} \mathrm{mm} \mathbf{~ I g}$ and a cathetertration pesk-to-peak gradient $\geq 75 \mathrm{~mm}$ Hg. The cross-hatched box on the lower lefi represents the data from 3 potients who ind not require interveation and who had a Doppler mean gradont <17 mm Hs and catheferization peak-to-peak gradient $<50 \mathrm{~mm}$ Hg. The intermediate group in the dotled area represeints 18 childen, 11 of whom required intervention and 7 who id not. In this intermed ate group, the mean gradient wes between 17 and $27 \mathrm{~mm} \mathrm{Hg}$, and the catheterivetion peakto-pesk gradient was between 50 and $75 \mathrm{~mm} \mathrm{Hg}$. In these chlidren, the Doppler mean gradient alone did not predict the need for intervention. Several data points on this figure are saperimposed. peak-to-peak pressure gradients using linear regression analysis ( $\mathrm{p}<0.05$ indicated a significant correlation).

\section{RESULTS}

The data obtained from the review of the Doppler examination, the cardiac catheterization report and the medical records are listed in Table I. Using linear regression analysis, the Doppler mean and peak instantaneous pressure gradients were compared to the catheterization peak-to-peak gradients (Figure 1). Significant correlations were found for both relations and the correlation coefficients were similar ( $r=0.74$ for mean gradient and 0.73 for peak instantaneous gradient).

For each patient, the Doppler mean gradient, the catheterization peak-to-peak gradient and the need for intervention are compared in Figure 2. All patients with a Doppler mean gradient $>27 \mathrm{~mm} \mathrm{Hg}$ required intervention. This group included 12 children, all of whom had a catheterization peak-to-peak gradient of $\geq 75 \mathrm{~mm}$ $\mathrm{Hg}$. All patients with Doppler mean gradients $<17 \mathrm{~mm}$ $\mathrm{Hg}$ did not require intervention. This group included 3 patients, all with peak-to-peak gradients $<50 \mathrm{~mm} \mathrm{Hg}$. The remaining 18 patients with Doppler mean gradients between 17 and $27 \mathrm{~mm} \mathrm{Hg}$ comprised an intermediate group in whom the Doppler mean gradient alone did not predict the need for intervention. In this group, catheterization peak-to-peak pressure gradients were between 50 and $75 \mathrm{~mm} \mathrm{Hg}$.

The sensitivity and specificity of a given Doppler mean gradient for predicting intervention were calculated from a chi-square table (Figure 3). As Figure 3 shows, a Doppler mean gradient $>27 \mathrm{~mm} \mathrm{Hg}$ predicts the need for intervention with $100 \%$ specificity (no false positives); however, the sensitivity is low at $52 \%$ (11 false negatives). In other words, there were 11 patients

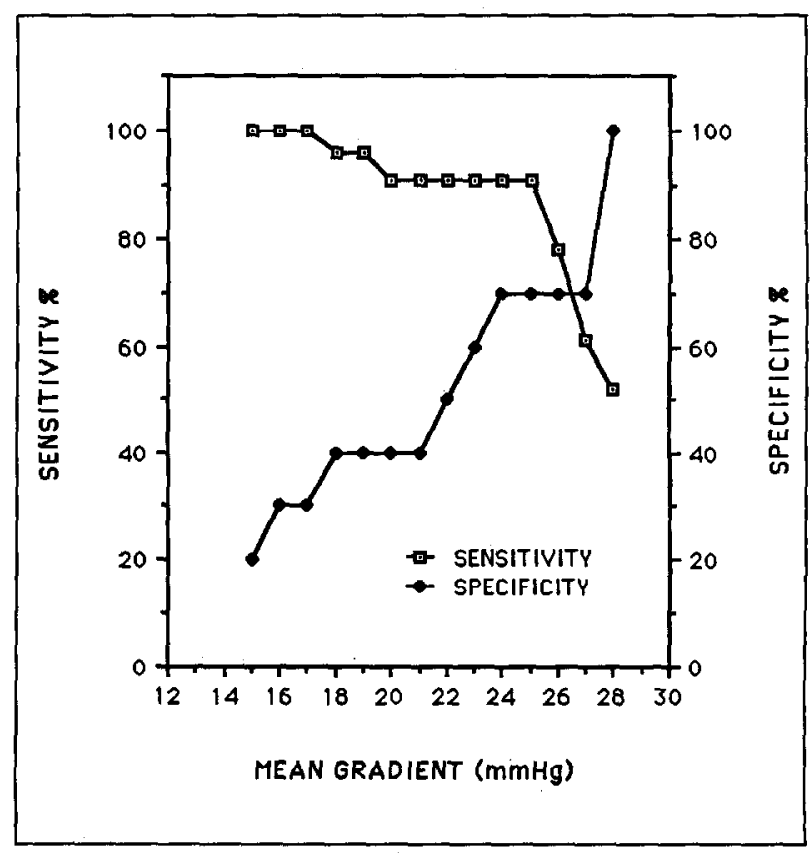

FGURE 3. The sensittity and specificity for predicting the need for intervention in children with aortic stenods. 


\begin{tabular}{|c|c|c|c|c|c|c|c|c|c|}
\hline \multirow[b]{2}{*}{ Pt } & \multirow[b]{2}{*}{$\begin{array}{l}\text { Age } \\
\text { (yrs) }\end{array}$} & \multicolumn{2}{|c|}{ Doppler Gradients } & \multicolumn{3}{|c|}{ Catheterization Data } & \multirow[b]{2}{*}{$\mathrm{S}$} & \multirow[b]{2}{*}{ ETा } & \multirow[b]{2}{*}{ I } \\
\hline & & $\begin{array}{l}\text { Mean } \\
(\mathrm{mm} \mathrm{Hg})\end{array}$ & $\begin{array}{l}\text { Peak } \\
(\mathrm{mm} \mathrm{Hg})\end{array}$ & $\begin{array}{l}\text { PPG } \\
(\mathrm{mm} \mathrm{Hg})\end{array}$ & $\begin{array}{l}\mathrm{Cl} \\
\text { (Liters/ } \\
\mathrm{min} / \mathrm{m}^{2} \text { ) }\end{array}$ & $\begin{array}{l}\text { LV S/D } \\
(\mathrm{mm} \mathrm{Hg})\end{array}$ & & & \\
\hline 1 & 0.25 & 27 & 88 & 42 & 4.4 & $132 / 18$ & 0 & - & 0 \\
\hline 2 & 0.25 & 32 & 94 & 92 & - & $168 / 16$ & 0 & - & + \\
\hline 3 & 0.33 & 46 & 93 & 102 & 3.1 & $162 / 12$ & + & - & + \\
\hline 4 & 0.75 & 44 & 109 & 106 & 2.5 & $178 / 15$ & + & - & + \\
\hline 5 & 1.5 & 27 & 77 & 104 & 5.5 & $176 / 18$ & 0 & - & + \\
\hline 6 & 1.9 & 23 & 68 & 38 & 4.2 & $148 / 12$ & 0 & - & 0 \\
\hline 7 & 2 & 32 & 103 & 109 & 2.3 & $191 / 15$ & 0 & - & + \\
\hline 8 & 4.5 & 36 & 111 & 80 & 3.9 & $184 / 12$ & 0 & - & + \\
\hline 9 & 4.7 & 26 & 72 & 68 & 4 & $176 / 20$ & + & - & + \\
\hline 10 & 4.8 & 51 & 102 & 82 & 3.9 & $178 / 12$ & 0 & - & + \\
\hline 11 & 5 & 25 & 73 & 55 & 3.9 & $160 / 17$ & + & + & + \\
\hline 12 & 6 & 15 & 26 & 27 & 4 & $122 / 20$ & 0 & - & 0 \\
\hline 13 & 6.5 & 26 & 75 & 76 & 4.5 & $198 / 15$ & + & - & + \\
\hline 14 & 7 & 13 & 95 & 43 & 3.5 & $131 / 10$ & + & 0 & 0 \\
\hline 15 & 8.5 & 27 & 86 & 50 & 4 & $170 / 14$ & 0 & 0 & 0 \\
\hline 16 & 11 & 26 & 74 & 80 & 3.7 & $210 / 22$ & + & + & + \\
\hline 17 & 11 & 50 & 132 & 120 & 4.3 & $220 / 10$ & 0 & + & + \\
\hline 18 & 12 & 28 & 72 & 84 & 3.9 & $194 / 17$ & + & + & + \\
\hline 19 & 12 & 19 & 62 & 50 & 4.9 & $170 / 10$ & + & + & + \\
\hline 20 & 14 & 26 & 74 & 75 & 2.9 & $175 / 13$ & 0 & + & + \\
\hline 21 & 14 & 36 & 67 & 76 & 4.9 & $188 / 13$ & 0 & + & + \\
\hline 22 & 14 & 12 & 43 & 47 & 4 & $160 / 10$ & 0 & + & 0 \\
\hline 23 & 14 & 25 & 70 & 80 & 4.5 & $200 / 12$ & 0 & + & + \\
\hline 24 & 15 & 17 & 49 & 63 & 3.4 & $173 / 14$ & + & + & + \\
\hline 25 & 15 & 27 & 73 & 64 & 4.9 & $200 / 22$ & 0 & + & + \\
\hline 26 & 16 & 33 & 86 & 85 & 2.9 & $200 / 16$ & 0 & - & + \\
\hline 27 & 16 & 17 & 41 & 30 & 3.2 & $120 / 12$ & 0 & 0 & 0 \\
\hline 28 & 16 & 32 & 82 & 80 & 3.7 & $190 / 15$ & + & - & + \\
\hline 29 & 16 & 44 & 116 & 100 & 4 & $220 / 18$ & 0 & + & + \\
\hline 30 & 17 & 27 & 69 & 34 & 3.6 & $180 / 20$ & 0 & + & 0 \\
\hline 31 & 18 & 22 & 36 & 25 & 3 & $143 / 12$ & + & 0 & 0 \\
\hline 32 & 18 & 21 & 58 & 50 & 3.2 & $145 / 8$ & 0 & 0 & 0 \\
\hline 33 & 20 & 25 & 64 & 78 & 2.8 & $192 / 9$ & 0 & + & + \\
\hline
\end{tabular}

who required intervention and who were not detected by this criterion alone. If a Doppler mean gradient of $>24$ $\mathrm{mm} \mathrm{Hg}$ is used as the criterion for intervention, then the sensitivity increases dramatically to $91 \%$ ( 2 false negatives), but the specificity decreases to $70 \%$ (3 false positives). At lower Doppler mean gradients, sensitivity does not increase appreciably while specificity drops off considerably.

To improve our ability to predict the need for intervention in patients with a Doppler mean gradient between 17 and $27 \mathrm{~mm} \mathrm{Hg}$, we combined symptoms and an abnormal exercise treadmill test result with the Doppler mean gradient as criteria for intervention (Figure 4). Thus, if the criterion for intervention is a Doppler mean gradient $>27 \mathrm{~mm} \mathrm{Hg}$ or a Doppler mean gradient between 17 and $27 \mathrm{~mm} \mathrm{Hg}$ in the presence of symptoms or an abnormal exercise treadmill test result, then the sensitivity and specificity improve considerably (Figure 4). A Doppler mean gradient $\geq 17 \mathrm{~mm} \mathrm{Hg}$ in the presence of symptoms or an abnormal exercise treadmill test result predicts the need for intervention with $96 \%$ sensitivity ( 1 false negative) and $80 \%$ specificity ( 2 false positives).
These combined criteria did not predict the need for intervention correctly in 3 of the 33 study patients (Table II). One patient who required intervention was not detected. This patient had a borderline Doppler mean gradient of $27 \mathrm{~mm} \mathrm{Hg}$ with no symptoms and a catheterization peak-to-peak gradient of $104 \mathrm{~mm} \mathrm{Hg}$. Two patients were predicted as requiring intervention but did not receive it. One patient had a Doppler mean gradient of $27 \mathrm{~mm} \mathrm{Hg}$, a catheterization peak-to-peak gradient of $50 \mathrm{~mm} \mathrm{Hg}$ and an abnormal blood pressure response to exercise. Although the patient had criteria for intervention, the decision was made not to intervene. The second patient had syncope with a Doppler mean gradient of $22 \mathrm{~mm} \mathrm{Hg}$ and a catheterization peak-to-peak gradient of $25 \mathrm{~mm} \mathrm{Hg}$. It is likely that symptoms in this patient were unrelated to AS.

\section{Discussion}

In children with AS, the peak-to-peak pressure gradient measured at cardiac catheterization is usually a reliable indicator of the severity of the obstruction and the need for intervention. This pressure gradient is affected not only by the severity of the AS but also by the 
TABLE II Patients Not Correctly Identified by Doppler Mean Gradient $>27 \mathrm{~mm} \mathrm{Hg}$ or Between 17 and $27 \mathrm{~mm} \mathrm{Hg}$ with Symptoms or Abnormal Exercise Treadmill Test

\begin{tabular}{|c|c|c|c|c|c|}
\hline Pt & Outcome & $\begin{array}{l}\text { Doppler } \\
\text { Mean } \\
\text { Gradient } \\
\text { (mm Hg) }\end{array}$ & $\begin{array}{l}\text { Catheterization } \\
\text { Gradient } \\
(\mathrm{mm} \mathrm{Hg})\end{array}$ & ETT & Symptoms \\
\hline 1 & False - & 27 & 104 & - & 0 \\
\hline 2 & False + & 27 & 50 & Abn & 0 \\
\hline 3 & False + & 22 & 25 & $\mathbf{N I}$ & Syncope \\
\hline
\end{tabular}

amount of transvalvular flow. Children with AS, unlike adults, do not usually have diminished or increased left ventricular stroke volume; therefore, this and other pressure gradients can be used to predict accurately the degree of AS.

Transvalvular pressure gradients can be measured reliably by Doppler echocardiography as well. The peak instantaneous pressure gradient across the aortic valve can be calculated from the Doppler recording of the peak transaortic flow velocity using the simplified Bernoulli equation..$^{1-4}$ When simultaneous Doppler and cardiac catheterization studies are performed, the Doppler-predicted peak instantaneous pressure gradient correlates closely with that measured at cardiac catheterization. ${ }^{6,13}$ However, the peak instantaneous pressure gradient is not routinely measured at cardiac catheterization as an indicator of the need for intervention; therefore, the clinician has no suitable reference standard to which the Doppler peak instantaneous pressure gradient can be compared. The Doppler-predicted mean pressure gradient is directly comparable to mean pres-

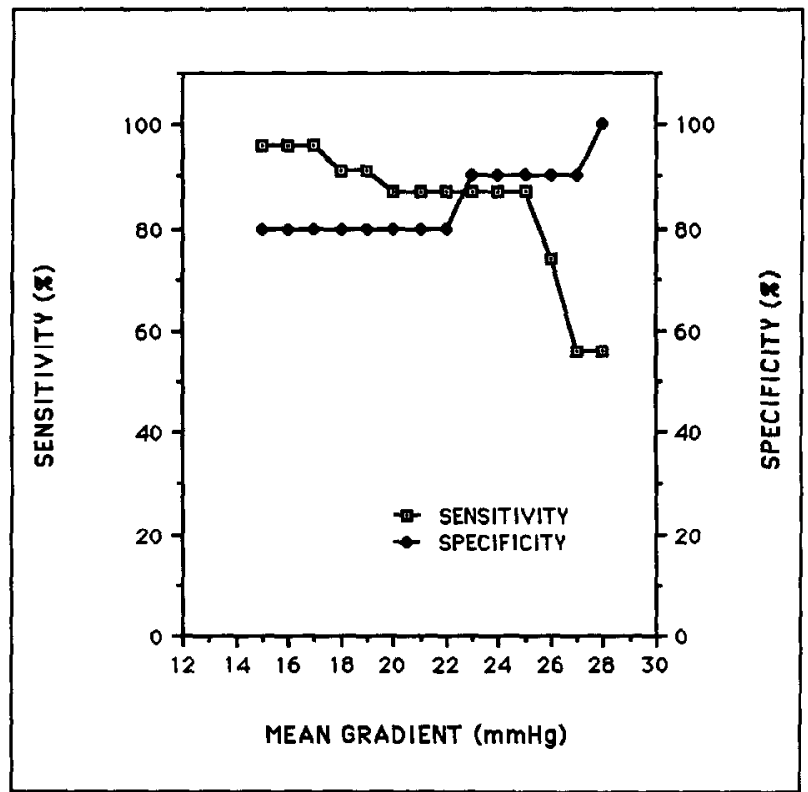

FGQURE 4. The conalitulty and spoctifity for prodicting the need for intervention in children with acortic stenoats when the criterion for intervention is a Dopplar masn gredient $>27 \mathrm{~mm}$ Hs or a Doppler mean gradient between 17 and $27 \mathrm{~mm} \mathrm{~Hz} \mathrm{~m}$ the presence of symptoms or an abnormal exercise treadtnil teat reant. sure gradient measured at cardiac catheterization. ${ }^{7-12}$ Little information, however, is available in pediatric patients relating the mean pressure gradient to the severity of stenosis and the need for intervention. In this study, we found that all children with a Doppler mean gradient $>27 \mathrm{~mm} \mathrm{Hg}$ had a catheterization peak-to-peak pressure gradient $\geq 75 \mathrm{~mm} \mathrm{Hg}$ and, therefore, required intervention. All children with a Doppler mean gradient $<17 \mathrm{~mm} \mathrm{Hg}$ had a catheterization peak-to-peak pressure gradient $<50 \mathrm{~mm} \mathrm{Hg}$ and did not require intervention. Children with Doppler mean gradients between 17 and $27 \mathrm{~mm} \mathrm{Hg}$ comprised an intermediate group with peak-to-peak pressure gradients between 50 and $75 \mathrm{~mm}$ Hg. In this group, the Doppler mean gradient alone was not sufficient to predict the need for intervention; however, the presence of symptoms or an abnormal exercise test provided the additional necessary information for an accurate noninvasive assessment of the severity of the AS.

In this study, a Doppler mean gradient $>27 \mathrm{~mm} \mathrm{Hg}$ or between 17 and $27 \mathrm{~mm} \mathrm{Hg}$ in the presence of symptoms or an abnormal exercise treadmill test predicted the need for intervention with $96 \%$ sensitivity ( 1 false negative) and $80 \%$ specificity ( 2 false positives). One of the false-positive patients had clinical criteria for intervention and did not receive it. Management decisions in any individual patient cannot always adhere to a rigid set of guidelines. This patient represents a deviation from the guidelines rather than an erroneous test result. In the other false-positive patient, intervention was predicted on the basis of a Doppler mean gradient in the intermediate range in the presence of symptoms (syncope). However, symptoms in this patient were unrelated to the AS and no intervention was necessary. The remaining false-negative patient had a borderline Doppler mean gradient of $27 \mathrm{~mm} \mathrm{Hg}$, no symptoms and a catheterization peak-to-peak gradient of $104 \mathrm{~mm} \mathrm{Hg}$. The large discrepancy between the Doppler mean gradient and the catheterization peak-to-peak gradient in this patient probably stems from failure during the Doppler examination to record the jet velocities at an acceptable intercept angle.

The Doppler-predicted mean and peak instantaneous pressure gradients correlated significantly with the peak-to-peak pressure gradient measured at cardiac catheterization $(r=0.74$ for mean gradient and 0.73 for peak instantaneous gradient). In several previously reported studies, $2,3,5$ correlation coefficients have been higher. We believe that the correlation coefficients in our study were lower for the following reasons: (1) the Doppler and catheterization measurements were not made simultaneously ${ }^{13}$; (2) no patient was excluded because of the quality of the Doppler tracing; and (3) the study was performed retrospectively rather than prospectively, which influences who does the examination and how hard the examiner works to obtain the perfect Doppler tracing.

Acknowlodgment: We wish to thank Steven Spillan and Kathlene Chmielewski, CMA, for editorial assistance in the preparation of this manuscript. 


\section{REFERENCES}

1. Hatle L, Angelsen BA, Tromsdal A. Non-invasive assessment of aortic stenosis by Doppler ultrasound. Br Heart J 1980;43:284-292.

2. Young JB, Quinones MA, Waggoner AD, Miller RR. Diagnosis and quantification of aortic stenosis with pulsed Doppler echocardiography. Am J Cardiol 1980;45:987-944

3. Oliveira LC, Sahn DJ, Valdes-Cruz LM, Allen HD, Goldberg SJ, Grenadier E, Barron JV. Prediction of the severity of left ventricular outflow tract obstruction by quantitative two-dimensional echocardiographic Doppler studies. Circulation 1983,68:348-354.

4. Berger M, Berdoff RL, Gallerstein PE, Goldberg E. Evaluation of aortic stenosis by continuous wave Doppler ultrasound. JACC 1984;3:150-156.

5. Smith MD, Dawson PL, Elion JL, Booth DC, Handshoe R, Kwan OL, Earle GF, DeMaria AN. Correlation of continuous wave Doppler velocities with cardiac catheterization gradients: an experimental model of aortic stenosis. JACC 1985,6:1306-1314

6. Currie PJ, Hagler DJ, Seward JB, Reeder GS, Fyfe DA, Bove AA, Tajik AJ. Instantaneous pressure gradient: a simultaneous Doppler and dual catheter correlative study. JACC 1986,7:800-806.

7. Agatston AS, Chengot M, Rao A, Hildner F, Samet P. Doppler diagnosis of valvular aortic stenosis in patients over 60 years of age. Am J Cardiol 1985:56:106-109.

8. Otto CM, Pearlman AS, Comess KA, Reamer RP, Janko CL, Huntsman LL Determination of the stenotic aortic valve area in adults using Doppler echocardiography. JACC 1986:7:509-517.

9. Yeager M, Yock PG, Popp RL. Comparison of Doppler-derived pressure gradient to that determined at cardiac catheterization in adults with aortic valve stenosis: implications for management. Am J Cardiol 1986;57.644-648.

10. Oh JK, Taliercio CP, Holmes DR, Jr, Reeder GS, Baily KR, Seward JB, Tajik AJ. Prediction of the severity of aortic stenosis by Doppler aortic valve area determination: prospective Doppler-catheterization correlation in 100 patients. JACC 1988;11:1227 1234.

11. Fan P-H, Kapur KK, Nanda NC. Color-guided Doppler echocardiographic assessment of aortic valve stenosis. JACC 1988;12:441-449.

12. Nishimura RA, Holmes DR, Jr, Reeder GS, Orsulak TA, Bresnahan JF, Istrup DM, Tajik AJ. Doppler evaluation of results of percutaneous aortic balloon valvuloplasty in calcific aortic stenosis. Circulation 1988,78:791-799.

13. Currie PJ, Seward JB, Reeder GS, Vlietstra RE, Bresnahan DR, Bresnahan JF, Smith HC, Hagler DJ, Tajik AJ. Continuous-wave Doppler echocardiographic assessment of severity of calcific aortic stenosis: a simultaneous Doppler-catheter correlative study in 100 adult patients. Circulation 1985;71:1162-1169. 